\title{
Snake Venomics Display: An online toolbox for visualization of snake venomics data
}

Dam, Søren Helweg; Friis, Rasmus U.W.; Petersen, Søren D.; Martos-Esteban, Andrea; Laustsen, Andreas $\mathrm{H}$.

\section{Published in:}

Toxicon

Link to article, DOI:

10.1016/j.toxicon.2018.07.019

Publication date:

2018

Document Version

Peer reviewed version

Link back to DTU Orbit

Citation (APA):

Dam, S. H., Friis, R. U. W., Petersen, S. D., Martos-Esteban, A., \& Laustsen, A. H. (2018). Snake Venomics Display: An online toolbox for visualization of snake venomics data. Toxicon, 152, 60-64.

https://doi.org/10.1016/j.toxicon.2018.07.019

\section{General rights}

Copyright and moral rights for the publications made accessible in the public portal are retained by the authors and/or other copyright owners and it is a condition of accessing publications that users recognise and abide by the legal requirements associated with these rights.

- Users may download and print one copy of any publication from the public portal for the purpose of private study or research.

- You may not further distribute the material or use it for any profit-making activity or commercial gain

- You may freely distribute the URL identifying the publication in the public portal 
1 Snake Venomics Display: An online toolbox for visualization of snake

4 Søren H. Dam¹ ${ }^{1}$, Rasmus U. W. Friis ${ }^{1}$, Søren D. Petersen², Andrea Martos-Esteban ${ }^{1}$, Andreas H.

Laustsen $^{1 *}$

6

Lyngby, Denmark

${ }^{2}$ Novo Nordisk Foundation Center for Biosustainability, Technical University of Denmark, Kgs.

Address for correspondence:

15

16

17

18

19

20

21

22

23
Dr. Andreas H. Laustsen

Associate Professor

Department of Biotechnology and Biomedicine

Technical University of Denmark

DK-2800 Kgs. Lyngby, Denmark

*ahola@bio.dtu.dk 


\section{Abstract}

25 With the introduction of powerful mass spectrometry equipment into the field of snake venom 26 proteomics, a large body of venomics data is accumulating. To allow for better comparison between venom compositions from different snake species and to provide an online database containing this data, we devised the Venomics Display toolbox for visualization of venomics data on linear scales. This toolbox is freely available to be used online at https://tropicalpharmacology.com/tools/snakevenomics-display/ and allows researchers to visualize venomics data in a Relative Abundance (\%)

31 visualization mode and in an Absolute Abundance (mg) visualization mode, the latter taking venom

32 yields into account. The curated venomics data for all snake species included in this database is also made available in a downloadable Excel file format. The Venomics Display toolbox represents a

34 simple way of handling venomics data, which is better suited for large data sets of venom compositions from multiple snake species.

Keywords: Venomics; Snake venom; Venom proteomics; Snake Venomics Display; Visualization of venomics data, Snake venomics 
Venom proteomics has gained increasing attention in the last decade due to scientific interests in venom compositions, venom/toxin evolution, and elucidation of pathophysiological mechanisms of envenomings, as well as technological advances within proteomics and mass spectrometry that have allowed researchers to perform ever more in-depth investigations. In addition to venomics (Calvete, 2014), new fields have recently emerged, such as quantitative venomics, antivenomics, and functional venomics/toxicovenomics (Calvete, 2010; Calvete et al., 2017, 2009; Calvete and Lomonte, 2015; Laustsen, 2016; Laustsen et al., 2015a, 2015b; Lomonte and Calvete, 2017). Particularly within snake venomics, this has created a wealth of data that allows researchers to obtain a deeper understanding of venom function(s), evolutionary strategies for defense and prey subduction, and for developing improved antivenoms (Laustsen, n.d.; Laustsen et al., 2015a). However, with increasing complexity, a need for better structure and visualization of data presents itself. Inspired by how DNA and protein sequences are often visualized by linear alignment, and to alleviate the need for a database containing snake venomics data (Tasoulis and Isbister, 2017), we gathered all reported snake venomics data and created an Open Access visualization toolbox that is freely available to be used online. This toolbox, coined Snake Venomics Display, may help researchers obtain an overview of venom compositions within snake families, genera, and species, as well as guide scientist and physicians to quickly determine which toxin families are abundant in a given snakebite. This may be useful in the interpretation of large sets of venomics data, and it may aid the development of new antivenoms. Additionally, we have made all the venomics data included in our online database freely available in an Excel format by direct download via a link in the Snake Venomics Display toolbox. 


\section{Methods}

\section{Data collection}

Data on toxin abundances was retrieved for 232 venom proteomes and for 10 venom gland transcriptomes (identified by a superscripted asterix in the snake name) from 143 snake species characterized in 103 original articles reviewed by Calvete (Calvete, 2013), Laustsen et al. (Laustsen et al., 2016), and Tasoulis \& Isbister(Tasoulis and Isbister, 2017), and an online database was created. Abundances for trace toxins were consistently set to the maximum value of the interval (e.g. an abundance reported as $<0.1 \%$ was set to $0.1 \%$ in our database). Venom yields were obtained from http://snakedatabase.org/ for the 83 species for which this information was available. In cases where the total venom abundance (in \%) exceeded 100\%, the data was normalized. All included venomics data is available in an Excel format by direct download in the Snake Venomics Display toolbox.

\section{Building the program}

The program was designed using the WordPress plugin: "wpDataTables". This plugin is widely used for its table-generation feature, and it possesses highly customizable charting functionalities used for the Snake Venomics Display toolbox. The venomics database described above was loaded into the plugin, and filters were specified with user-friendliness and functionality in mind. The data was then loaded in the plugin's "HighCharts" rendering software to display an interactive horizontal bar chart. Important functions, like responsiveness and setting the chart to follow the filter, were activated. A color scheme was manually defined so that colors express the protein family of the toxins. Visual specifications for the chart, such as units for axes and numbers, size, and credits link, were set, and the chart was embedded on the webpage. The same procedure was executed for both Snake Venomics Display visualization modes (Relative Abundance and Absolute Abundance). 


\section{How to use the Snake Venomics Display toolbox}

Upon loading the Snake Venomics Display toolbox at https://tropicalpharmacology.com/tools/snake-

venomics-display/, a small description of the two currently available visualization modes (Relative

Abundance and Absolute Abundance) is displayed together with buttons to access these modes

(Figure 1). By clicking on one of these buttons, the user can use a tool in the Snake Venomics Display

toolbox. The toolbox is by default filtered to display all venomics data available. However, the displayed snakes can be modified using the filtering system below the chart. Here, seven settings can be modified: "Snake" (name in Latin), "Common names" (in English), "Genus", "Family", "Country", http://www.toxinology.com/. Searching for a country in the "Country" filter will display all snakes endemic in that specific country. "Family" allows the user to define which snake family to look at. snakes with a given common name. The common names have been acquired from http://www.toxinology.com/. The "Snake" filter is modified by typing the desired snake species or genus using Latin names, while "Common names" is modified by typing the desired common name of a species in English. "Country" and "Region" are applied similarly by typing the desired region and/or country. "Genus" and "Family" are specified using a drop-down menu. To remove the current

108 filter and display all snakes in the database, the "CLEAR FILTERS" button is clicked. By clicking on the snake name, the user will be taken to the PubMed entry of the original publication from which 
110 the data was collected. Finally, an "add data by request" button is provided to allow users to submit

111 their own published venomics data to help keep the database up to date.

113 Relative venom compositions

114 The Snake Venomics Display toolbox enables easy comparison of snake venoms by linear 115 visualization of venomics data. By selecting the Relative Abundance Display, the user will be able to 116 compare relative venom compositions (toxin family abundances expressed as percent of whole 117 venom) for families, genera, and species of snakes from different regions of the world. This can be 118 useful for quickly obtaining an overview of which toxin families are dominant in a given snake venom 119 and how this compares with closely and/or distantly related species. It is envisioned that this may be 120 particularly useful for researchers who wish to compare venoms from many snake species in relation 121 to snake venom evolution.

As an example, the relative abundance of toxin families present in venoms from the Bitis genus can be seen in Figure 2. From this visualization, it quickly becomes evident that the Bitis venoms share a similar relative venom composition, although Bitis caudalis distinguishes itself by having a much higher proportion $\mathrm{PLA}_{2} \mathrm{~S}$ (purple). genus, the venom compositions for four selected Naja venoms are clearly more similar in terms of relative abundance of toxin families (Figure 3). However, as these snake species are reported to

129 deliver very different venom yields when milked, the clinical manifestations may differ between bites

130 from different species within the genus. To visualize venom compositions in closer relation to the 131 possible medical impact that a bite from a given snake species may have, the Absolute Abundance visualization mode was developed. 


\section{Absolute venom compositions}

135 The Absolute Abundance visualization mode was developed to take venom yields into account when 136 displaying venomics data. This represents a simple way of observing venom compositions, which 137 may possibly provide a slightly more reliable indication of which toxin families are most medically 138 relevant for a given envenoming case. As an example of this visualization mode, the same venoms 139 from the Bitis genus are displayed in Figure 4. Here, it quickly becomes evident that even though $B$. 140 caudalis venom is dominated by $\mathrm{PLA}_{2} \mathrm{~s}$, this toxin family is more likely to have an actual medical 141 impact in bites from the Bitis gabonica species due to the insignificant venom yields delivered by $B$. 142 caudalis and the high venom yields delivered by B. gabonica. Similarly, a very different picture emerges when comparing the previously selected

144 Naja venoms in the Absolute Abundance visualization mode (Figure 5). Although bites from all four snake species should be handled as critical medical emergencies, it seems evident that severe bites from Naja mossambica can be more serious than bites from the other species.

A word of caution needs to be highlighted. Although the Absolute Abundance visualization mode may seem useful for evaluating the medical impact of bites from different snake species, this should in no case be used uncritically. Snakebites differ significantly, not only between species, but also between different specimens, with larger specimens being able to deliver larger

151 amounts of venom in a bite. The Absolute Abundance visualization mode should thus mainly be used 152 to provide a general overview of the possible severity of bites from different snake species 153 conceivably in introductory educational settings.

\section{Conclusions}

155 Snake Venomics Display is a toolbox for linear visualization of snake venomics data, which allows

156 for better comparison of large sets of snake venom proteomes. This toolbox will be continuously 157 improved for user-friendliness, as well as it will be updated with venomics data as this becomes 
158 available. We invite researchers in the field to submit both such data as well as comments and

159 feedback, which we can use to make the Snake Venomics Display toolbox even more useful.

160

\section{Acknowledgments}

162 We thank Professor Juan Calvete, Instituto Biomedicina Valencia (Spain) for fruitful scientific 163 discussion. Thanks also go to the Novo Nordisk Foundation for financial support 164 (NNF16OC0019248).

165

166 Author contributions

167 A.H.L. conceived the study. S.D.P. collected the data. S.H.D., R.U.W.F., and A.M.E. designed and 168 developed the program. S.H.D. and R.U.W.F. analyzed the case data presented in the manuscript. 169 A.H.L., S.D.P., S.H.D., and R.U.W.F. wrote the manuscript. All authors reviewed the manuscript. 


\section{References}

Calvete, J.J., 2014. Next-generation snake venomics: protein-locus resolution through venom proteome decomplexation. Expert Rev. Proteomics 11, 315-329. https://doi.org/10.1586/14789450.2014.900447

Calvete, J.J., 2013. Snake venomics: From the inventory of toxins to biology. Toxicon, Special Issue: Toxins: from Threats to Benefits20th Meeting of the French Society of Toxinology (SFET) 75, 44-62. https://doi.org/10.1016/j.toxicon.2013.03.020

Calvete, J.J., 2010. Antivenomics and venom phenotyping: A marriage of convenience to address the performance and range of clinical use of antivenoms. Toxicon, Highlights in Toxinology: Biodiversity in Toxins - Tools for Biological Research and Drug Development 56, 1284-1291. https://doi.org/10.1016/j.toxicon.2009.12.015

Calvete, J.J., Lomonte, B., 2015. A bright future for integrative venomics. Toxicon, Omic perspectives of the toxin universe 107, Part B, 159-162. https://doi.org/10.1016/j.toxicon.2015.10.024

Calvete, J.J., Petras, D., Calderón-Celis, F., Lomonte, B., Encinar, J.R., Sanz-Medel, A., 2017. Protein-species quantitative venomics: looking through a crystal ball. J. Venom. Anim. Toxins Trop. Dis. 23, 27. https://doi.org/10.1186/s40409-017-0116-9

Calvete, J.J., Sanz, L., Angulo, Y., Lomonte, B., Gutiérrez, J.M., 2009. Venoms, venomics, antivenomics. FEBS Lett., Prague Special Issue: Functional Genomics and Proteomics 583, 1736-1743. https://doi.org/10.1016/j.febslet.2009.03.029

Laustsen, A.H., 2016. Recombinant antivenoms, 1st ed. University of Copenhagen, Copenhagen, Denmark.

Laustsen, A.H., n.d. Guiding recombinant antivenom development by omics technologies. New Biotechnol. https://doi.org/10.1016/j.nbt.2017.05.005

Laustsen, A.H., Engmark, M., Milbo, C., Johannesen, J., Lomonte, B., Gutiérrez, J.M., Lohse, B., 2016. From Fangs to Pharmacology: The Future of Snakebite Envenoming Therapy. Curr. Pharm. Des. 22, 5270-5293. https://doi.org/10.2174/1381612822666160623073438

Laustsen, A.H., Lohse, B., Lomonte, B., Engmark, M., Gutiérrez, J.M., 2015a. Selecting key toxins for focused development of elapid snake antivenoms and inhibitors guided by a Toxicity Score. Toxicon 104, 43-45. https://doi.org/10.1016/j.toxicon.2015.07.334

Laustsen, A.H., Lomonte, B., Lohse, B., Fernández, J., Gutiérrez, J.M., 2015b. Unveiling the nature of black mamba (Dendroaspis polylepis) venom through venomics and antivenom immunoprofiling: Identification of key toxin targets for antivenom development. J. Proteomics 119, 126-142. https://doi.org/10.1016/j.jprot.2015.02.002

Lomonte, B., Calvete, J.J., 2017. Strategies in "snake venomics" aiming at an integrative view of compositional, functional, and immunological characteristics of venoms. J. Venom. Anim. Toxins Trop. Dis. 23. https://doi.org/10.1186/s40409-017-0117-8

Tasoulis, T., Isbister, G.K., 2017. A Review and Database of Snake Venom Proteomes. Toxins 9. https://doi.org/10.3390/toxins9090290 


\section{Figure legends}

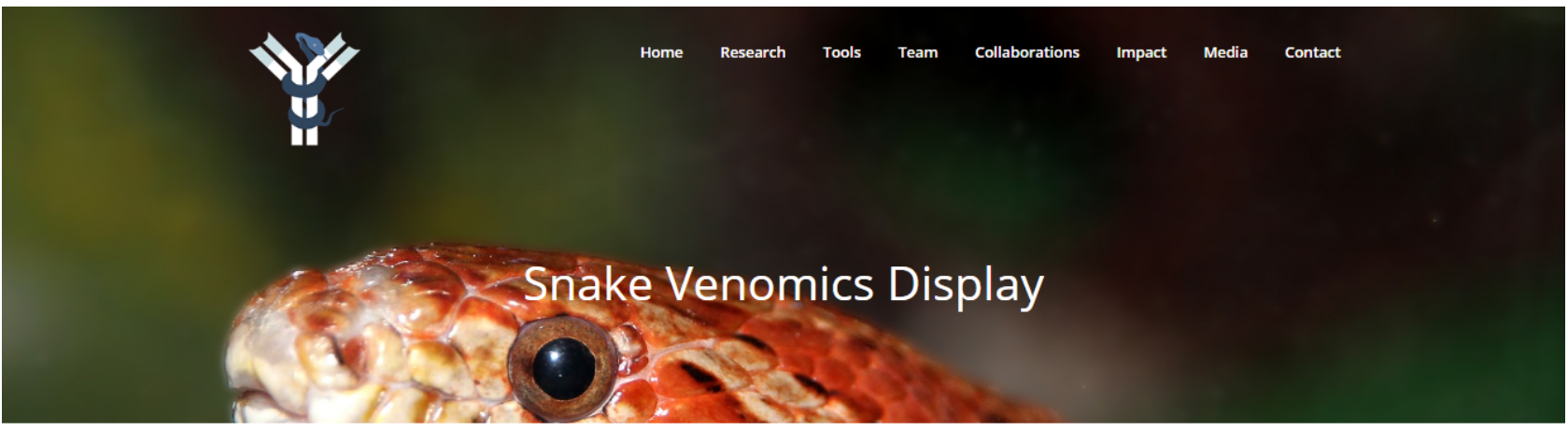

The severity of a snakebite envenomation is related to the amount of venom injected and its composition of toxins. We have developed a linear visualization tool for better display of venomics data. Our tools, Relative Abundance Display and Absolute Abundance Display, are developed to help guide venom and antivenom researchers with an intuitive overview of venom compositions, and the amount of toxins likely to be injected by various venomous snakes. In addition, the user can go to the original research article clicking on the snake name. Relative Abundance Display and Absolute Abundance Display may also help clinicians in predicting development of envenoming symptoms by providing a quick and quantitative ovenview of snake venom proteomes. Absolute Abundance Display also facilitates a fast identification of

the toxins with the highest medical relevance for a given venom.

214 Figure 1. Initial view upon loading the Snake Venomics Display toolbox

215 (https://tropicalpharmacology.com/tools/snake-venomics-display/).

Bitis arietans

Bitis caudalis

Bitis gabonica

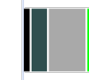

Bitis nasicornis

Bitis gabonica rhinoceros
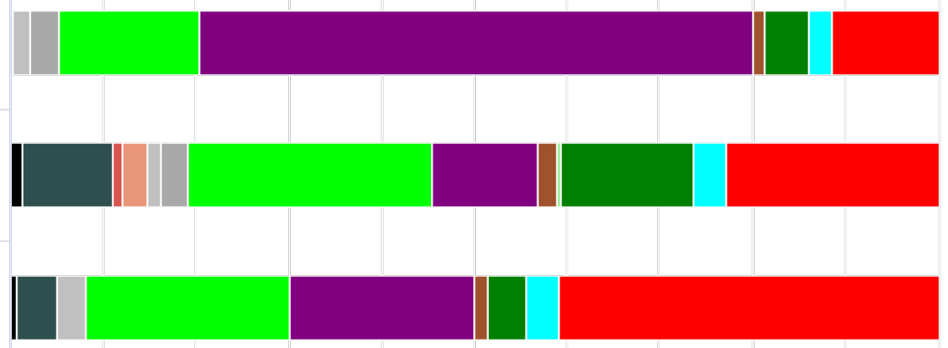

$0 \%$
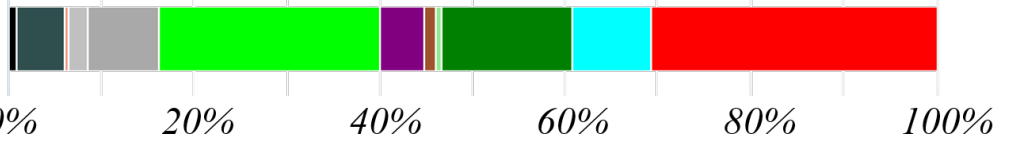

Venom yield

- SVMP

- $P L A_{2}$

- $S P$

CTL

Cystatin

Disintegrin 
218 Figure 2. Display of the relative composition of selected venoms from the Bitis genus. Abbreviations:

219 SVMP: Snake Venom MetalloProteinase. PLA2: PhosphoLipase A2. SP: Serine Proteinase. CTL: C220 Type Lectin.

Naja katiensis

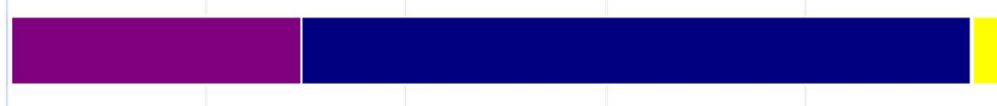

Naja mossambica

Naja nigricollis

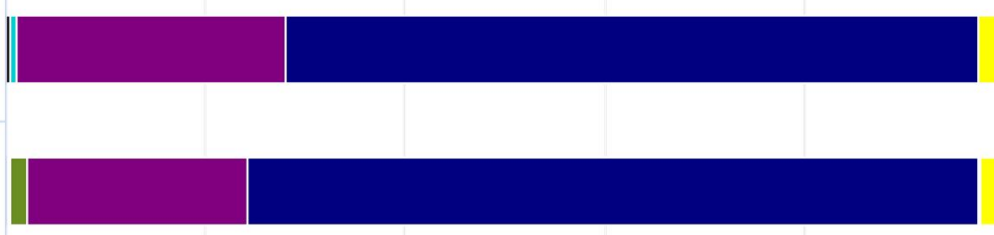

Naja pallida

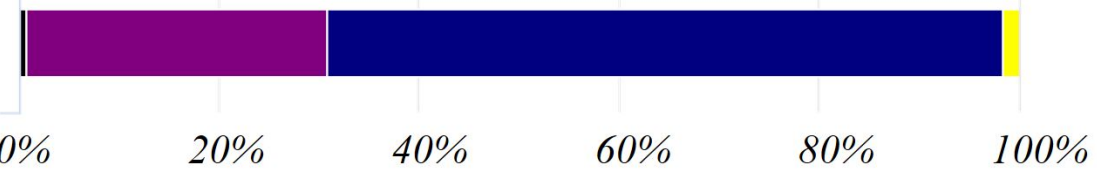

Venom yield

223 Figure 3. Display of the relative compositions of selected venoms from the Naja genus. 224 Abbreviations: SVMP: Snake Venom Metalloproteinases. PLA2: Phospholipases A2. 3FTx: Three225 Finger Toxins. 


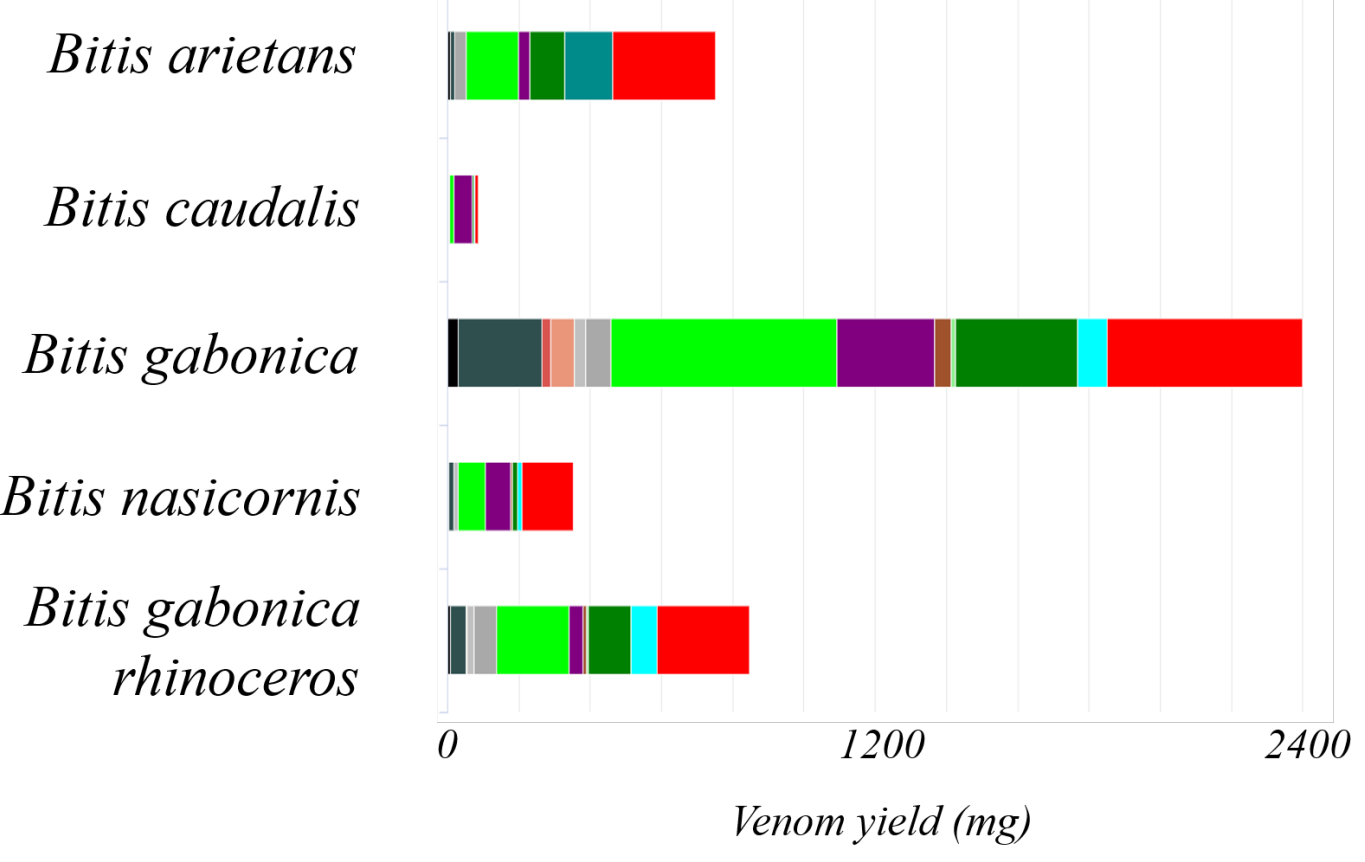

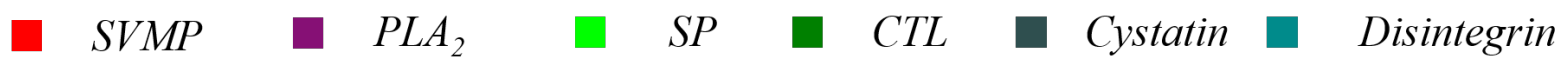

228 Figure 4. Display of the absolute compositions of selected venoms from the Bitis genus.

229 Abbreviations: SVMP: Snake Venom Metalloproteinase. PLA 2 : Phospholipases A2. SP: Serine 230 Proteinases. CTL: C-Type Lectins. 
Naja katiensis

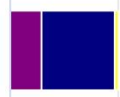

Naja mossambica

Naja nigricollis

Naja pallida
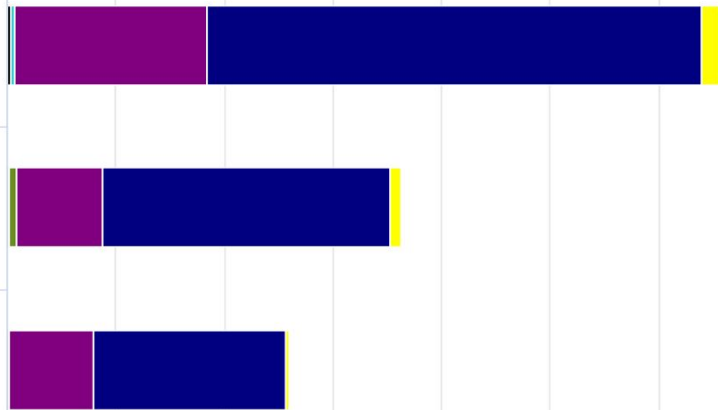

0

200

400

600

Venom yield $(\mathrm{mg})$

233 Figure 5. Display of the absolute compositions of selected venoms from the Naja genus.

234 Abbreviations: SVMP: Snake Venom Metalloproteinases. PLA 2 : Phospholipases $\mathrm{A}_{2}$. 3FTx: Three235 Finger Toxins. 